\title{
OSCILLATIONS OF FREEZING FRONT IN EXTERNAL ENCLOSURES
}

\author{
Natalija Parfentjeva', Oleg Samarin ${ }^{2}$, Sabina Paulauskaité ${ }^{3}$ \\ 1. ${ }^{2}$ Moscow's State University of Civil Engineering, Jaroslavskoje st. 26, 129337 Moscow, Russia \\ ${ }^{3}$ Vilnius Gediminas Technical University, Sauletekio al. 11, LT-2040 Vilnius, Lithuania \\ 'E-mail:natasha_@dataforce2E-mail: samarin1@mtu-net.ru ${ }^{3} E$-mail: spaul@ap.vtu.lt
}

Received 26 Sept 2002; accepted 16 Jan 2003

\begin{abstract}
An approximate procedure is proposed for calculating of the temperature field in a one-layer wall under harmonic variations of the external air temperature with regard to phase transitions of moisture within the wall. The trend of the freezing front displacement within the wall is researched as time goes on depending on relative thermal stability of the wall material and average thermal lag of the freezing layer. The engineering formulae are obtained for quantitative estimation of the front displacement offering determination of the peak value of its waves and their time lag with respect to the external temperature oscillations. The estimation of accuracy of the proposed calculation procedure is carried out and the limits of its suitability are detected. It has been shown that the error of the procedure falls in the limits of initial data under the magnitudes of the air temperature and thermal properties of employed materials meeting with in practice if the freezing front lays beyond the bounds of the violent temperature oscillation layer.
\end{abstract}

Keywords: external enclosures, temperature field, moisture, phase transition, calculation procedure.

\section{Introduction}

In the presented paper the distribution of temperature waves in an one-layer outside enclosure is considered in view of phase transitions of moisture contained in the enclosure material. The purpose of this research was to develop a technique for accounting temperature fluctuations in an enclosure under specified conditions. This is important, as any protecting design comprises a certain quantity of moisture that is absorbed from air of a premise and during a testing phase transitions while in service of an enclosure. A number of investigations on moisture behaviour in building envelope are provided yet, especially in northern countries [1,2]. In Lithuania with a concern about the durability of outer layer at aggressiveness of climatic changes the research results are presented in $[3,4]$.

At the same time, until now an account of an enclosure's thermal stability was not taken into consideration. This will be shown below as well as the significant error because of high inertial properties of the phase border. Besides, this research on the behaviour of a freezing front plays an important role in the study of a real thermal and a humidity regime of the enclosure and the questions that are connected with the durability of protecting designs.

\section{The research objectives}

For the research realisation we will solve a problem of the distribution of temperatures in a section of a wall depending on a time assumption, where the temperature field is one-dimensional, the temperature of the outside air changes by the harmonic law, and the temperature of the internal air is constant.

\section{The research method}

In this case the mathematical formulation of the task looks as follows: it is necessary to solve the thermal conductivity equation [5]

$$
\partial T / \partial \tau=a \partial^{2} T / \partial x^{2}
$$

at boundary and entry conditions:

$x=0: T_{e}=T_{a v}+A \cos (\omega \tau) ;(\lambda \partial \mathrm{T} / \partial x)_{x=+0}=\left(\left.T\right|_{x=+0}-T_{e}\right) \alpha_{e} ;$ $x=\delta: T_{i}=$ const $;-(\lambda \partial \mathrm{T} / \partial x)_{x=\delta-0}=\left(\left.T\right|_{x=\delta-0}-T_{i}\right) \alpha_{i}$. (2)

Here $T$ is the temperature of a wall, $X-$ the spatial coordinate, $\delta$ - the thickness of a wall, $\lambda$ and $a$ - accordingly factors of the thermal and temperature conductivity of the wall material; $\alpha_{e}$ and $\alpha_{i}$ - factors of convective heat exchange between a wall and according to the external and internal air; $T_{e}$ and $T_{i}$ - temperatures of the outside and internal air. $A, \omega$ and $T_{a v}$ are the amplitude, frequency of fluctuations and the average magnitude of $T_{e}$ for the period.

Therefore on the border of the unit of phases (at the freezing front) the following conditions take place [6]: 


$$
\begin{gathered}
x=x_{F}:\left.T\right|_{x=x F-0}=\eta_{x=x F+0}=0 \\
\rho r_{m} \partial x_{F} / \partial \tau=(\lambda \partial T / \partial x)_{x=x F-0}-(\lambda \partial \mathrm{T} / \partial x)_{x=x F+0} . \\
\text { (the Stephan condition) }
\end{gathered}
$$

Where $X_{F}$ is the co-ordinate $X$ of the phase border of the moisture contained in the wall material, $r_{m}$ is the specific melting heat of water and $\rho$ is the volumetric humidity of the wall material (weight of the moisture on volume unit of the enclosure).

At the task stating in the form (1)-(3) we neglected the moisture flow through the wall material and processes of evaporation/condensation on its surfaces because of their small influence on the basic process. Also, we considered that the moisture is distributed in the thicker wall uniformly. Nevertheless, even in such a case the analytical decision of the task is extremely difficult and inconvenient for practical use [7]. Therefore we shall offer some simplifications allowing the receiving of an acceptable result for engineering practice.

First of all, we consider that the temperature waves are distributed outside only up to the freezing front, ie to $X_{F}$ and further the distribution of the temperature corresponds to a stationary one for each time moment. To make such an assumption, the value of $r_{m}$ of water $(334 \mathrm{~kJ} / \mathrm{kg}$ ) allows, owing to the fact that the freezing front has a large thermal inertia. More precisely, the magnitude of criterion $c A / r_{m}$, where $c$ is the specific heat capacity of the wall material determining the ratio between heat accumulation in a file of the enclosure and at the freezing front, for the usual values of $A$ (near 5 degrees) does not exceed 0,02 , that lays within the limits of usual accuracy of engineering accounts. Therefore a specified simplification is admitted.

Besides, we will take advantage of the linearity and uniformity of the Fourier-Kirchhoff equation (1) and properties of boundary tasks with harmonic boundary conditions [5] and we present a task as a superposition of two situations:

1) stationary:

$x=0: T_{e}=T_{a v} ;(\lambda \partial \mathrm{T} / \partial x)_{x=+0}=\left(\left.T\right|_{x=+0}-T_{a v}\right) \alpha_{e} ;$

$x=\delta: T_{i}=$ const $;-(\lambda \partial \mathrm{T} / \partial x)_{x=\delta-0}=\left(\left.T\right|_{x=\delta-0}-T_{i}\right) \alpha_{i}$.

When for the average value of $X_{F}$ for the period is received then:

$$
x_{F . a v}=\frac{\lambda / \alpha_{i}+\delta \lambda_{r} t_{e} / \lambda_{l} t_{i}+\lambda_{r} / \alpha_{e}}{\lambda_{r} t_{e} / \lambda_{l} t_{i}-1}+\lambda_{r} / \alpha_{e} .
$$

Where $\lambda_{l}$ and $\lambda_{r}$ are according to thermal conductivity factors of wall sites saturated with a liquid and firm moisture;

2) non-stationary:

$$
\begin{aligned}
& x=0: T_{e}=A \cos (\omega \tau) ;(\lambda \partial \mathrm{T} / \partial x)_{x=+0}=\left(\left.T\right|_{x=+0}-T_{e}\right) \alpha_{e} ; \\
& x=\delta: T_{i}=0 ;-(\lambda \partial \mathrm{T} / \partial x)_{x=\delta-0}=\left.T\right|_{x=\delta-0} \alpha_{i} .
\end{aligned}
$$

The condition (3) on the phase border is maintained. Now we shall make one more simplification: we assume, that in the 1st approximation $X_{F}=X_{F . a v}=$ const, and we receive a task about the distribution of temperature waves in a flat wall without phase transformations with mixed boundary conditions of the 3rd and 1st type:

$$
\begin{aligned}
& x=0: T_{e}=A \cos (\omega \tau) ;(\lambda \partial \mathrm{T} / \partial x)_{x=+0}=\left(\left.T\right|_{x=+0}-T_{e}\right) \alpha_{e^{j}} \\
& x=x_{F}:\left.T\right|_{x=x F-0}=\left.T\right|_{x=x F+0}=0 .
\end{aligned}
$$

The scheme of the temperature waves distribution in an external enclosure under these conditions is shown in Fig where $A_{x F}$ is the amplitude of $X_{F}$.

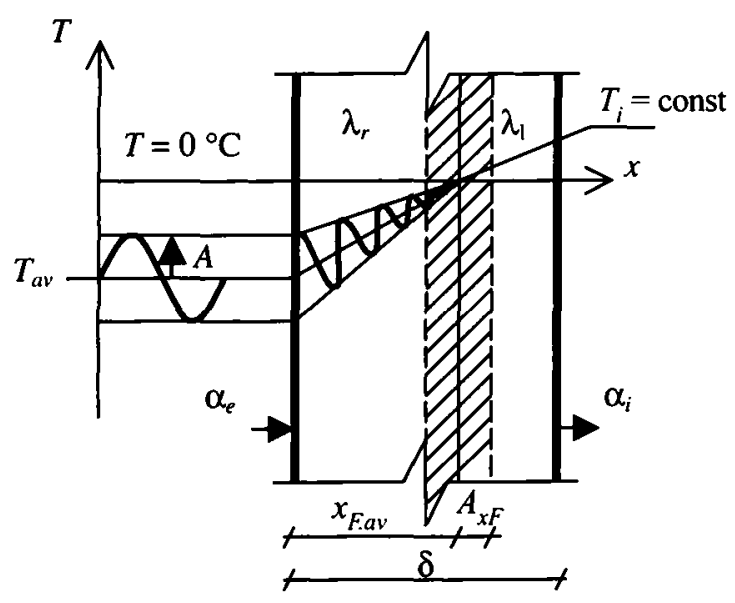

The scheme of the temperature waves distribution in an external enclosure

For the solution of this task we shall take advantage of a method of complex functions [8], and for complex temperature, the statement of a task will be written down as follows:

$$
\begin{aligned}
& x=0: \Theta_{e}=A \exp (i \omega \tau) ;(\lambda \partial \Theta / \partial x)_{x=+0}=\left(\left.\Theta\right|_{x=+0}-\Theta_{e}\right) \alpha_{e} ; \\
& x=x_{F}:\left.\Theta\right|_{x=x F-0}=\left.\Theta\right|_{x=x F+0}=0 .
\end{aligned}
$$

Method of variable division gives the solution as

$$
\Theta=\frac{A \exp (i \omega \tau) \sin \left[\left(x-x_{F}\right) \sqrt{i \omega / a}\right]}{\left[1-(\lambda c \rho \omega)_{r} / \alpha_{e}^{2}\right]^{/ 2} \sin \left[x_{F} \sqrt{i \omega / a}+\operatorname{arctg}\left(\sqrt{i \lambda c \rho \omega_{r} / \alpha_{e}}\right)\right]},
$$

where $c$ is a specific heat capacity of wall material, $\mathrm{kJ} / \mathrm{kg} \mathrm{K}$. Since $(\lambda c \rho \omega)_{r}^{1 / 2}=S_{r}$ is the heat admittance factor of a wall material saturated with a firm moisture, and $X(\omega / a)^{12}=D_{x}$ is the thermal inertia of a wall layer by thickness $X$ [9], after some transformations, the complex solution is the result:

$$
\Theta=\frac{A \exp (i \omega \tau) \sin \left[\left(D_{x}-D_{F}\right) \sqrt{i}\right]}{\left(1-s_{r}^{2} / \alpha_{e}^{2}\right)^{1 / 2} \sin \left[D_{F} \sqrt{i}+\operatorname{arctg}\left(\sqrt{i} \cdot s_{r} / \alpha_{e}\right)\right]} .
$$

Where $D_{F}=X_{F}(\omega / a)^{1 / 2}$ is the thermal inertia of a wall layer up to the freezing front. Now it is possible to take advantage of the Stephan condition (3) and to determine with its help the change of a situation of the (14) freezing front, depending on the time. With the as- 
sumptions $\Theta=0+0 i$ for $X>X_{F}$, the Stephan condition in this case is the following:

$$
\rho r_{m} \partial x_{F} / \partial \tau=(\lambda \partial \Theta / \partial x)_{x=x F-0} .
$$

Substituting in (10) expressions for $\Theta$ from (9), after some simplifications we can receive:

$$
\begin{aligned}
& \rho r_{m} \partial x_{F} / \partial \tau= \\
& \frac{A \exp (i \omega \tau) s_{r} \sqrt{i}}{\left(1-s_{r}^{2} / \alpha_{e}^{2}\right)^{1 / 2} \sin \left[D_{F} \sqrt{i}+\operatorname{arctg}\left(\sqrt{i} \cdot s_{r} / \alpha_{e}\right)\right]} .
\end{aligned}
$$

After the integration we find the required expression for $X_{F}$ in a complex kind:

$$
\frac{X_{F}(\tau)=}{\rho r_{m} i \omega\left(1-s_{r}^{2} / \alpha_{e}^{2}\right)^{1 / 2} \sin \left[D_{F} \sqrt{i}+\operatorname{arctg}\left(\sqrt{i} \cdot s_{r} / \alpha_{e}\right)\right]} .
$$

Where the integration constant was determined from a condition of equality to zero of both parts of expression (12), on the average for the period.

Allocating now real parts from (12), we receive a final result as some function $X_{F}=X_{F}(\tau)$; then we will find the specified value of $\Theta$ by substituting (9) for $X$ expressions $X^{*} X_{F \cdot a v} / X_{F}(\tau)$. After that the real part of $\Theta$ in sum with the stationary solution gives the required distribution of the temperature on the section of a wall.

On the basis of the offered algorithm, a computer program was made, with the help of which the calculations were carried out. The analysis of the received results shows their qualitative affinity to a picture of distribution of temperature waves under usual conditions, ie without an account of the phase's transformations. Besides, it is obvious that at the harmonic change of $T_{e}$ the value of $X_{F}$ changes harmonically too, with a certain shift of phases in relation to $T_{e}$, that also corresponds to the physics of the investigated phenomenon.

Let us estimate now the error of the approximate solution (9)-(12). For this purpose we shall determine the jump of amplitude of the thermal flow fluctuation at the freezing front, connected with its inertial properties. Then we shall compare it with the amplitude of a thermal flow causing changes in the time of the allocation of $X_{F}$ and, with the occurrence of a non-stationary component of a temperature field in the field of a wall with $T>0$. This is we neglected at the conclusion (9). It is possible to find this amplitude from the expression (11). Using the solution [9] for the one-sided freezing of a flat wall and transforming it to a kind which takes into account the harmonic character of the outside temperature change, we shall receive:

$$
\Delta\left(A_{q}\right)=\left[\frac{2 A \lambda \rho r_{m} \omega}{\exp \left(-D_{F} / \sqrt{2}\right)}\right]^{1 / 2} .
$$

If we divide the left and right parts of expressions (13) and (11) apart, it is possible to find the relation of the specified additional thermal flow to a flow causing the freezing front displacement and to determine a degree of influence of the front that is moving a temperature field in an external part of the enclosure. After some transformations to the module of the specified attitude we find:

$$
\delta_{q}=\left[\frac{2 r_{m}\left(1-s_{r}^{2} / \alpha_{e}^{2}\right)}{A c \cdot \exp \left(-D_{F} / \sqrt{2}\right)}\right]^{1 / 2} .
$$

Therefore in the formula (14) there is a dimensionless parameter $A c / r_{m}$. It is obvious that the more $\delta_{q}$, the more will be the relative thermal inertia of the freezing front, and the more effective it detains fluctuations of a thermal flow. Thus the magnitude of $\delta_{q}$ can be accepted as the criterion determining the applicability of the received decision from the point of view of an allowable error of engineering accounting. Accordingly, at a quantitative estimation, it appears that the error of the decision (9-12) makes, in relative expression, no more than 10-15\% (return size to $\delta_{q}$ ).

Therefore the complication of the decision for a temperature field by corrective action which is taking into account the displacement of the freezing front, practically is inexpedient if we mean that accuracy of the initial data $(\lambda, S$ etc) and especially the allowable mistake of the engineering account makes $5-10 \%$, ie it is less than the error (12). This means, that it is possible to be limited to the specification of the value of $X_{F}$ at calculation $D_{F}$ as it is made in the note of the formula (12). It is actually enough at each time moment to carry out a scaling of a temperature field at the expense of the moving border with zero temperature without accounting the distortions which are brought in by the penetration of fluctuations of a thermal flow for freezing front.

\section{The research results}

The numerical realisation of the solutions (9)-(12) is feasible only with the help of a computer, as the analytical allocation of a material part from (9)-(12) results in bulky calculations. Therefore we shall carry out a research on the behaviour of the complex solution, depending on the parameters included in it for the reception of dependence and suitable for hand-operated calculations. First of all, we will address the expression $X_{F}$ ie it interests us to the highest degree. From (12) we can receive:

$$
X_{F}=\stackrel{A s_{r}}{\rho r_{m} \omega} \cdot f(\tau)+X_{F \cdot a v} .
$$

Where the complex $A s_{r} / \rho r_{m} \omega$ does not depend on $\tau, S_{r} / \alpha_{e}$ and $D_{F}$ and the function $f$ is the real part of expression

$$
\frac{\exp (i \omega \tau)-1}{i\left(1-s_{r}^{2} / \alpha_{e}^{2}\right)^{1 / 2} \sin \left[D_{F} \sqrt{i}+\operatorname{arctg}\left(\sqrt{i} \cdot s_{r} / \alpha_{e}\right)\right]}
$$


It is obvious that $f$ should be the harmonic function and we shall search for it as:

$$
f(\tau)=B\left(D_{F} ; s_{r} / \alpha_{e}\right) \cos (\omega \tau+\varphi) .
$$

The functions $B$ and $\varphi$ are defined on the basis of a numerical study of the behaviour of the function $f$ at various $S_{r} / \alpha_{e}$ and $D_{F}$ with the help of a special computer program. According to the results of this account, diagrams are constructed which can be already be used for practical calculations. The analysis of these diagrams shows their good conformity to the physics of the investigated process and allows one to look after the law of change of the amplitude and phase of fluctuations of the freezing front with its distance from an outside surface of a wall and with a change of heat exchange conditions [10]. Besides, the kind of curves received in a certain measure prompts the structure of the approximation formulas for $B$ and $\varphi$, which can be received for a zone "of regular fluctuations" (practically at $D_{F}>1$ ):

$$
\begin{aligned}
& B=1,85 \exp \left(-0,56 s_{r} / \alpha_{e}-D_{F} / \sqrt{2}\right) \\
& \varphi=2,33-D_{F} / \sqrt{2}-0,36 S_{r} / \alpha_{e} .
\end{aligned}
$$

The accuracy of the given dependence under the condition of $S_{r} / \alpha_{e}<1,2$, that is almost always observed in real conditions, it is not worse than $3-5 \%$. Besides, as the formulas (18) are received in a criteria kind, they are universal for a wide spectrum of magnitudes of set parameters.

\section{Example}

As an example, we can quote the results of calculation of the value $A_{x F}$ for the styrofoam thermal insulation layer with $\delta=0,2 \mathrm{~m}$ and magnitudes of its physical parameters [1 1] $c=1340 \mathrm{~J} / \mathrm{kg} \mathrm{K}, \lambda_{r}=0,052 \mathrm{~W} /(\mathrm{m} \mathrm{K})$, $\rho=10 \mathrm{~kg} / \mathrm{m}^{3}, S=0,82 \mathrm{~W} /\left(\mathrm{m}^{2} \mathrm{~K}\right)$ and $A=-5^{\circ} \mathrm{C}(24$-hour amplitude for Moscow) and for comparing the brick wall with $\delta=0,51 \mathrm{~m}, c=880 \mathrm{~J} / \mathrm{kg} \mathrm{K}, \lambda_{r}=0,81 \mathrm{~W} /(\mathrm{m} \mathrm{K})$, $\rho=36 \mathrm{~kg} / \mathrm{m}^{3}$ and $S=10,12 \mathrm{~W} /\left(\mathrm{m}^{2} \mathrm{~K}\right)$. Then using formulae (15)-(18) we obtain magnitudes $A_{x F}$ equal to 4,65 and $4,31 \mathrm{~mm}$ correspondingly. Calculation with the help of the computer using direct solution of the equation system (1) $-(3)$ by finite-differential scheme gives the results of 5,48 and $4,70 \mathrm{~mm}$, ie disagreement does not exceed $8-14 \%$, that is not more than the accuracy of initial information given in [11].

\section{Conclusions}

Finally for $X_{F}$ we can receive:

$$
X_{F}=\frac{A s_{r}}{\rho r_{m} \omega} \cdot B \cos (\omega \tau+\varphi)+X_{F . a v}
$$

Where $X_{F . a v}$ is calculated by the formula (5) and $B$ and $\varphi$ by the formulas (18), the above is true. Therefore with $D_{F}$ it is convenient to determine this as $X_{F} \cdot S_{r} / \lambda_{r}$.

By a further development of a technique it is also possible to receive the settlement ratio for $T$, as well as to apply the found dependence to a case of a multi-layer enclosure. The results can be used at accounting for the thermal stability of enclosures.

\section{References}

1. Cunningham, M. J. Moisture diffusion due to periodic moisture and temperature boundary conditions - an approximate steady analytical solution with non-constant diffusion coefficients. Journal of Building and Environment, Vol 27, No 3, 1992, p. 367-377.

2. De Freitas, V. P.; Brantes, V.; Rausse, P. Moisture migration in buildings walls - analysis of the interface phenomena. Journal of Building and Environment, Vol 31, No 2, 1996, p. 99-108.

3. Mačiulaitis, R. Frost resistance and durability of ceramic facade product (Fasadinès keramikos atsparumas šalčiui ir ilgaamžiškumas). Vilnius: Technika, 1996. 132 p. (in Lithuanian, English and Germany).

4. Barkauskas, V.; Stankevičius, V. Thermophysics of building envelope (Pastatı atitvaru šiluminè fizika). Kaunas: Technologija, 2000. 286 p. (in Lithuanian).

5. Sveshnikov, A. S.; Bogolyubov, A. N.; Kravtsov, V. N Lectures on mathematical physics (Лекции по математической физике). Moscow: Izd-vo MGU, 1993. 353 p. (in Russian).

6. Budaiwi, I.; El-Diasty, R. and Abdou, A. Modelling of moisture and thermal transient behavior of multi-layer noncavity wall. Journal of Building and Environment, Vol 34, 1999, p. 537-551.

7. Prusakov, G. M. Mathematical models and methods of the calculations of electronic computation machine (Математические модели и методы в расчетах на ЭВМ). Moscow: Nauka, 1993. 144 p. (in Russian).

8. Christian, J. E.; Kosny, J. Thermal performance and wall ratings. Journal ASHRAE, March 1996, p. 56-65.

9. Bogoslovsky, V. N. Thermophysics of buildings (Строительная теплофизика). Moscow: Vyschaja shkola, 1982. 416 p. (in Russian).

10. Cekanavičius, A. Moisture changing tendencies in walls additionally insulated from the inside. Civil Engineering (Statyba), Vol IV, No 3. Vilnius: Technika, 1998, p. $214-$ 218.

11. SniP II-3-79* Building Thermal Technology (Строительная теплотехника). Moscow: GUP CPP, 1998. 29 р. (in Russian). 